\title{
Design and Performance Measurement of Energy-based Acoustic Signal Detection with Autonomous Underwater Vehicles
}

\author{
Redouane Es-sadaoui ${ }^{1}$, Jamal Khallaayoune ${ }^{2}$ \\ Department of Electronic, Microwave and Optic \\ National Institute of Posts and Telecommunications \\ Rabat, Morocco
}

\begin{abstract}
The Autonomous Underwater Vehicles (AUVs) industry is still awaiting its Henry Ford to bring to the market solutions that are well adapted to the challenge of underwater exploration. This will certainly be done by the advent of small connected drones equipped with small sensors and embedded devices, allowing AUVs to operate in a coordinated swarm, at a unit price so affordable that we can consider deploying hundreds, or even thousands simultaneously, to be able to observe the ocean with an instrument of a size finally adapted to its immensity. The scope of this work is to build a high performance and lowcost embedded device easy to mount onboard small AUVs and implementing energy-based spectrum sensing algorithms in order to detect targets underwater using acoustic waves. The principle of design, hardware architecture and real-time implementation of the acoustic signal processing chain are described in this paper. Simulations and sea experiments have been conducted successfully and qualified the performance of the realized system to detect acoustic pings underwater depending on the signalto-noise ratio (SNR). Moreover, this paper proposes methods to improve the measured detection range and accuracy.
\end{abstract}

Keywords-Autonomous Underwater Vehicles (AUVs); acoustic signal processing; spectrum sensing; energy detection

\section{INTRODUCTION}

Many questions have been raised after the mission failure of BlueFin-21 (Fig. 1), a super-equipped AUV costing more than million dollars deployed in March 2014 by the U.S. Navy to spot black boxes of Boeing 777 of Malaysian Airlines flight MH370, crashed into the empty vastness of the southern Indian Ocean, killing all 239 passengers and crew onboard.

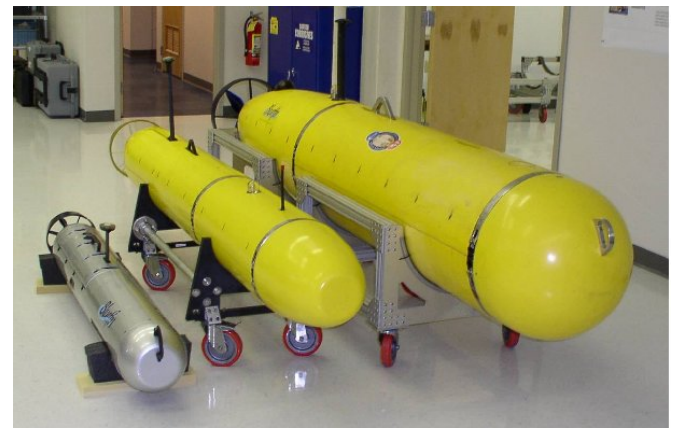

Fig. 1. From Left to Right : the Bluefin-21, Bluefin-12, and Bluefin-9 Autonomous Underwater Vehicles (AUVs) [1].

\author{
Tamara Brizard ${ }^{3}$ \\ Arkeocean SARL, 808 route de Tourrette Levens \\ 06790 Aspremont, France
}

To successfully capture the last acoustic signals transmitted by the black boxes, it would probably have been necessary to deploy a multitude of coordinated "swarm", forming a listening network deployed in the column of water over several square kilometers (see Fig. 2). But with AUVs costing tens or even hundreds of thousands of dollars, this concept is economically unrealistic.

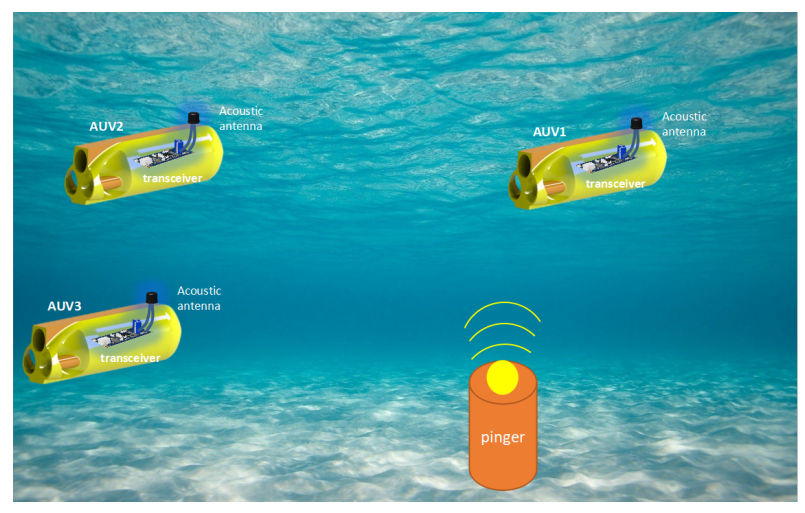

Fig. 2. Schematic Showing the Proposed Solution to Detect Targets Underwater using Small AUVs Operating in Swarm.

In recent years, as covered by [2], [3], oceanic based research is gradually growing across the globe trying to solve the major challenges of Underwater Acoustic Sensor Networks (UWASNs) like limited bandwidth, propagation, power constraint, localization and spectrum utilization, which is challenging compared to terrestrial applications due to the unavailability of GPS and to the unique physics of sensing in the marine environment where the search zone is immense, and the bad weather and rough seas hamper efforts to find objects underwater. However, the majority of research works are limited to theoretical studies and lack of real implementation with week contribution in the technical development of AUV industry.

The motivation behind this work arises from an absence of open architectures and easy-to-use solutions that give access to the physical variables of underwater acoustic systems, which, in turn, will allow the research community to design novel acoustic spectrum sensing algorithms. In this scope, the present paper presents a proof of concept and major steps required to 
design and develop a low volume acoustic device that can be installed onboard AUVs in order to detect targets underwater. These three main challenges will be investigated:

1) Addressing the "Underwater spectrum sensing challenge" : where the AUV should be able to sense acoustic signals transmitted by subsea targets.

2) Addressing the "Acoustic ranging challenge": providing the AUV with its relative distance to the transmitter of acoustic waves.

3) Addressing the "accurate detection challenge": Improving the detection performance especially in term of maximum range and Time-of-Arrival (ToA) measurement accuracy.

The paper is organized as follows. Section II reviews the acoustic spectrum sensing techniques. In Section III, the proposed methods are described by specifying the hardware architecture of the pinger and the transceiver, implementation of the acoustic signal processing chain and its simulation in MATLAB. Section IV presents the sea trial results and discusses solutions to improve the performance of the realized system. Conclusions are given at the end.

\section{Spectrum Sensing Methods ReView}

\section{A. Overview}

Above water, most autonomous systems rely on radio or spread-spectrum communications and global positioning. However, electromagnetic signals attenuate rapidly underwater, and therefore acoustic waves are needed because they are less attenuated and travel further in water, as detailed in [4] and [5]. The use of electromagnetism remains possible at "very low" or "extra low" frequencies, but their implementation to establish a subsea communication will require large antennas in general, incompatible with the small size of an AUV and will at most allow a few bits per second to be exchanged, which remains too limited for the operational use that is generally required with AUVs.

The terms "Spectrum sensing" and "detection" are nominally interchangeable. They denotes the process to identify the presence or absence of transmitters in a specific spectrum; see[6]. Spectrum sensing techniques can be classified into two main categories :

- $\quad$ Non-cooperative [7], [8]: where receivers must independently have the ability to determine the presence or absence of a transmitter in a specific spectrum.

- $\quad$ Cooperative [9], [10], [11]: where a group or network of receivers exchange information in order to enhance the detection performance.

This paper focuses on Non-cooperative category allowing each AUV to detect and recognize transmitters pinging in a specific spectrum. For reason of simplicity, the following comparison focuses on the two popular techniques (covered here [12]) that are matched-filtering and energy based detection.

\section{B. Matched Filtering based Detection}

The matched filtering based spectrum sensing technique, as detailed in [13], [14], uses the transmitted signal as a template to which the received signal is compared. The better match between the template and the received signal, is the greater amplitude of the matched filter's output. The received signal and pilot signal are convoluted and averaged over $N$ samples to obtain the matched filter decision statistic, which is then compared to the matched filter threshold $T_{M F}$ to get the sensing decision. $T_{M F}$ is calculated by:

$$
T_{M F}=\frac{1}{N} \sum_{n=1}^{N}\left(y(n) * x_{p}(n)\right)
$$

Where the received signal stream is denoted by $y(n)$, the known pilot signal is indicated by $x_{p}(n)$, and $N$ is the number of samples acquired in a sensing cycle Under $H 0$, the decision statistic, $T_{M F}$, is obtained by the averaged convolution of the Gaussian noise and the pilot signal. On the other hand, under $H 1, T_{M F}$ results from the convolution of the transmitted signal contaminated with the Gaussian noise and the pilot signal averaged over $N$ samples. The matched filter threshold, $\lambda_{M F}$ is taken from the "quiet time approach". Therefore, the noise is merely present in the received signal, $y(n)$. As a result, the matched filter threshold, $\lambda_{M F}$ is identical to the matched filter decision statistic, $T_{M F}$ during the quiet time period. If $\lambda_{M F}$ is determined, the binary hypothesis is given as:

$$
\begin{array}{ll}
H 0: & T_{M F}>\lambda_{M F} \\
H 1: & T_{M F}<\lambda_{M F}
\end{array}
$$

\section{Energy based Detection}

Energy detection (also called non-coherent detection; in reference to [15], [16]) is very popular and performed by simply comparing the output of the energy of the received signal energy with a predefined threshold. The decision statistic of an energy detector can be calculated from the squared magnitude of the FFT averaged over $N$ samples of the received signal. The detector output is the received signal energy as given by:

$$
T_{E D}=\sum_{n=0}^{N} y(n)^{2}
$$

Where $n=1 \ldots . N, N$ is the number of samples, and $y(n)$ is the received signal, and $T_{E D}$ denotes the energy of the received signal. The detection decision can be expressed as:

$$
\begin{array}{ll}
H 0: & T_{E D}>\lambda_{E D} \\
H 1: & T_{E D}<\lambda_{E D}
\end{array}
$$

Where $\lambda_{E D}$ denotes the energy detection threshold.

Our design will be based on energy detection mechanism which is easy to implement with moderate computational complexities and can be performed on both time and frequency domain. In addition, compared to the matched filtering, Energy detection does not require a prior information of the transmitted signal to operate. However, the detection threshold has to be selected carefully, as described in the next section.

\section{Detection Threshold}

The detection threshold is a decibel number that essentially incorporates the AUV acoustic transceiver ability to decide 
that a detection is made or not made. The detection process generally includes the following probabilities:

- The probability of detection (Pd): the probability that a signal is detected if it is present;

- 1-PD: the probability the signal will not be detected if it is present;

- The probability of false alarm (PFA): the probability that a signal is detected when it is not present;

- 1-PFA: the probability that the signal will not be detected when it is not present

As described in [17], [18], the detection threshold $D T$ with a Gaussian signal for a given Pfa:

$$
D T=10 \log \frac{Q^{-1} \cdot(P f a)-Q^{-1} \cdot(P d)^{2}}{2}
$$

Where $Q\left(\lambda_{E D}\right)=\operatorname{Proba}\left(Z>\lambda_{E D}\right)$ is the error function that validates the probability where a gaussian signal $Z$ of variance $\sigma$ and average $M$ reaches a threshold $\lambda_{E D}$.

The problem exposed here corresponds to a frequent question in a detection chain: how to adjust the threshold to control the false alarm rate? In the ideal case, the statistics of the noise alone are known and the threshold can be calculated (analytically or by simulation) from the desired false alarm rate, but in practice the characteristics of the noise are variable and it is therefore necessary to estimate the noise in permanently to have an correct estimation of the threshold. We consider here the case of a quadratic detection chain fed by Gaussian noise, the variance of which is known as detailed in [17], [18]. In this case the decision variable consists of the square of the input Gaussian variable and is therefore distributed according to an exponential law of density :

$$
p(x)=\frac{1}{2 \sigma^{2}} \cdot \exp \frac{-x}{2 \sigma^{2}} \cdot U(x)
$$

Where $U(x)$ designates the function which is worth 1 for $\mathrm{x}$ positive and 0 elsewhere (HEAVISIDE [19]). We can easily set the detection threshold from the desired $P f a$, for example, which $P f a=10^{-10}$ is not abnormal in an automatic detection device. We have :

$$
\operatorname{Proba}(X>\eta)=\exp \frac{-\eta}{2 \cdot \sigma^{2}}=10^{-10}
$$

Thus,

$$
\eta=10 \cdot \ln 10.2 \sigma^{2}
$$

We can therefore write the detection condition in the form:

$$
X-10 . \ln 10.2 . \sigma^{2}>0
$$

In practice $2 . \sigma^{2}$ is unknown and must therefore be replaced by an estimator noted $Y$. The detection condition then becomes:

$$
X-\lambda Y>0
$$

As result, the detection threshold will be biased and the value of $\lambda$ will be different from $10 . \ln 10$ due to the presence of $Y$ instead of $2 . \sigma^{2}$.

\section{Proposed Methods}

\section{A. Acoustic Pinger Design}

Refer to Fig. 3 for the next discussion. The acoustic pinger incorporates a PIC18 Micro-controller (MCU) [20] offering high computational performance at an economical price - with the addition of high-endurance, Flash program memory and introducing design enhancements that make these Microcontrollers a logical choice for many high performance, power sensitive applications. The Flash MCU outputs acoustic pulses in 8-bits digital format, the pulse is converted to an analog format by a Digital to Analog Converter (DAC), then amplified to high amplitude (around 24 volts) by means of an analog converter. A transformer is installed after the amplifier to raise the voltage of the acoustic pulse to a high amplitude voltage, which will attack an acoustic transducer ceramic that convert the electrical wave to an acoustic wave propagating in the water.

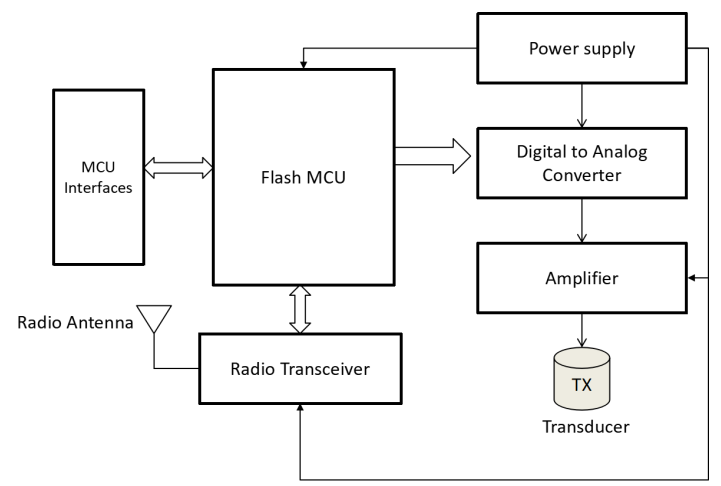

Fig. 3. Schematic Diagram Showing the Acoustic Pinger Architecture.

The DAC circuit was designed to interface between PIC MCU that are generating the acoustic pulse in digital format and the analog amplifier. It is rated as single, 8-bit, voltage out DAC that operates from a single 2.7 to 5.5 Volts supply and has a parallel microprocessor and DSP compatible interface with high speed registers and double buffered interface logic. Its on-chip precision output buffer allows the DAC output to swing rail to rail. The low power consumption of this part makes it ideally suited to portable battery operated equipment. The transmitter amplifier power supply is boosted to 24 Volts.

\section{B. Acoustic Transceiver Design}

The transceiver boards presented in Fig. 4 contains all the necessary circuits to condition and process signals received by the acoustic hydrophone. 


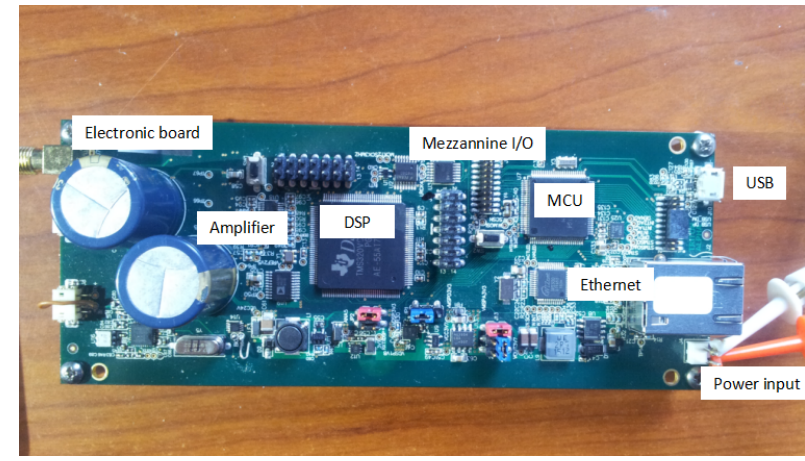

Fig. 4. Picture of the Acoustic Transceiver Board.

This section scrutinizes the blocks from Fig. 5, as well as their relations. The transceiver board can receive its power supply from the AUV and operate from 3.7 to 14 Volts. The power consumption of the board is about than $60 \mathrm{~mA}$ when fed under 12 Volts. The option to have an additional separate 24 Volts supply directly feeding the acoustic transmitter is also offered. With this option, the two large electrolytic capacitors are no longer necessary. A mezzanine for custom IOs or functions can be installed on the board as a MCU peripheral. many wireless on-chip modules were integrated through the mezzanine allowing the board to communicates via Radio $868 \mathrm{MHz} / 912 \mathrm{MHz}$, Lora, or WiFi.

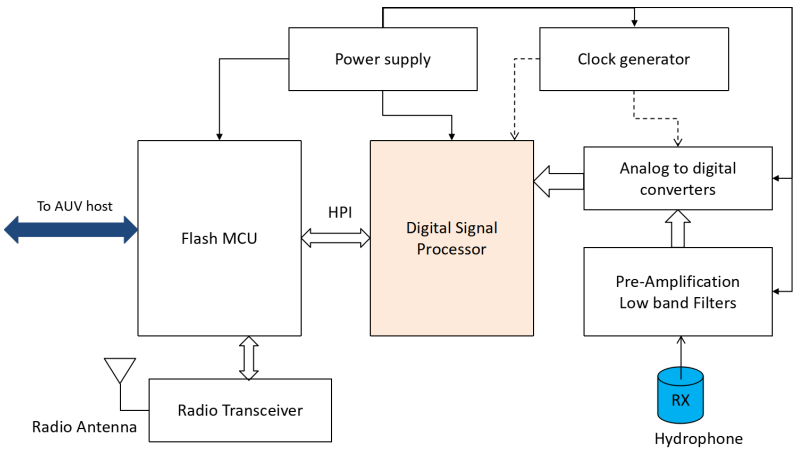

Fig. 5. Schematic Diagram Showing the Acoustic Transceiver Architecture.

The board incorporates a Texas Instruments TM320C5x Digital Signal Processor (DSP) [21] and MSP430F5xx MCU [22]. The DSP is a fixed-point processor based on an advanced modified Harvard architecture which provides an arithmetic logic unit (ALU) with a high degree of parallelism, applicationspecific hardware logic, on-chip memory, and additional onchip peripherals. The MSP430F5 family features a powerful 16-bit RISC CPU, 16-bit registers, and constant generators that contribute to maximum code efficiency. These MCUs include a high-performance 12-bit analog-to-digital converter (ADC), up to four universal serial communication interfaces, hardware multiplier, DMA, real time clock module with alarm capabilities, and I/O pins. Onboard the transceiver, this MCU manages the RF transceiver, the communication to the DSP using Host Port Interface (HPI), and communication to an external host and sensors. Furthermore, this MCU supports TI RTOS based software and open to custom applications. Inter-processor communications were implemented between the Flash MCU and the DSP based on the Host Port Interface link (HPI): A high speed parallel port through which the Flash MCU can directly access to DSP memory space.

Before being digitally processed, the hydrophone acoustic signals are first passed through a low pass filter and converted to digital format using a stereo ADC, that perform sampling, analog to digital conversion, and anti-alias filtering.

\section{Acoustic Chain}

As illustrated in Fig. 6, each received signal (a pure tone sine wave of constant frequency) is first amplified and filtered, then sampled through the ADC converter. The timeof-arrival of incoming pulses on the transceiver antenna is mainly measured by the DSP which implements an energy detector testing continuously if the pulse is present or not, and a high precision timer marking the detection timestamp. If we consider that the pinger and the transceiver share the same time reference [23], [24], the ToA of the incoming pulses can be estimated and the distance between the pinger and the transceiver will be determined.

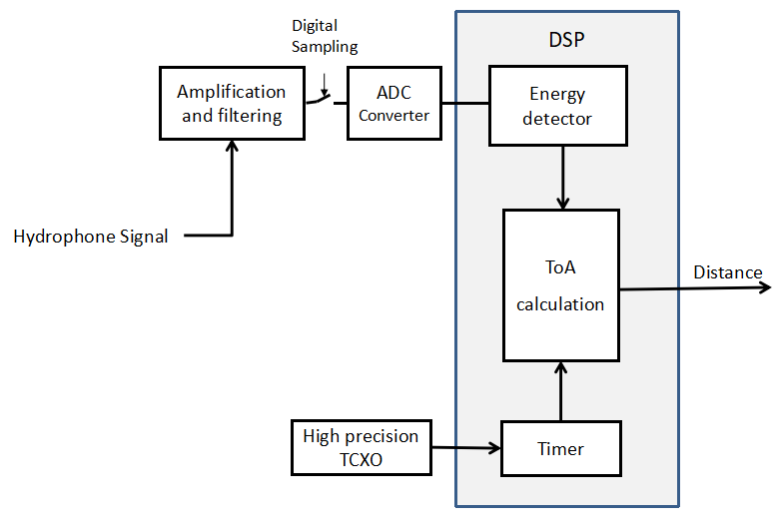

Fig. 6. Block Diagram Showing the Implementation of the Acoustic Signal Processing Chain. The Output is the Distance Between the Pinger and the Transceiver.

The transmitted pulse is a pure tone sine wave pulse, shaped in a balckman window: The pulse is combined from a blackman window multiplied by a sine wave. Equations (11) and (12) define respectively the mathematical formulas of the Blackman window and the transmitted pulse [25].

$$
w(k)=0.42-0.5 \cos \left(\frac{2 \pi \cdot k}{N-1}\right)+0.08 \cdot \cos \left(\frac{4 \pi \cdot k}{N-1}\right)
$$

Where $0 \leq k \leq M-1, \mathrm{~N}$ is the length of the blackman window. $\mathrm{M}$ is $\mathrm{N} / 2$ when $\mathrm{N}$ is even and $(\mathrm{N}+1) / 2$ when $\mathrm{N}$ is odd.

$$
S(k)=A \cdot \sin (2 \pi \cdot f / f e) \cdot w(k)
$$

Where A is the signal amplitude peak, $f$ is the signal frequency, fe is the sampling rate. The acoustic pulse form was simulated in Matlab and implemented in the real platform. The real output of the transmitter was measured and qualified by using an oscilloscope. Fig. 7 shows the used waveform, for a signal frequency of $22 \mathrm{KHz}$, a sampling frequency of $48 \mathrm{KHz}$ and a pulse length of 12 milliseconds. 


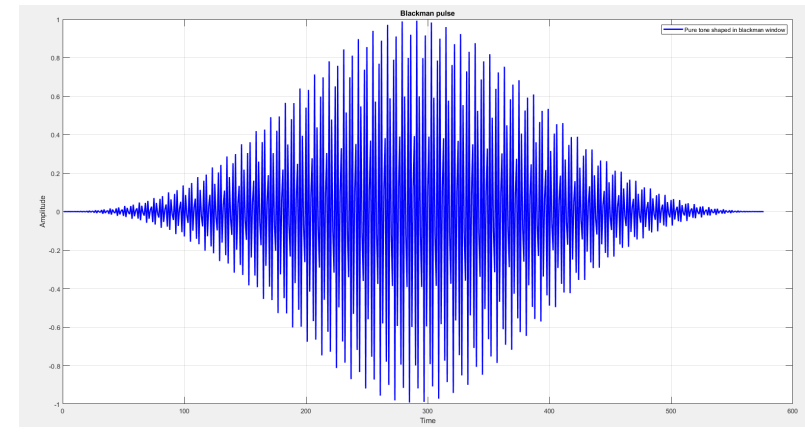

Fig. 7. Hydrophones Input Signals. Pure Tone Sine Wave Shaped in $12 \mathrm{~ms}$ blackman Window Without Noise. SNR $=100 \mathrm{~dB}$, Frequency $=22 \mathrm{kHz}$, Amplitude $=20 \mathrm{mV}$, Pulse width $=12 \mathrm{~ms}$, Sampling Rate $=48 \mathrm{kHz}$.

Horizontal Axis is the Time in Samples $(20.8 \mu \mathrm{s})$. Vertical Axis is the Amplitude in Volts.

This pulse was implemented on the Flash MCU of the acoustic pinger. Oscilloscope measurement of the pulse at the output of amplifier is illustrated in Fig. 8.

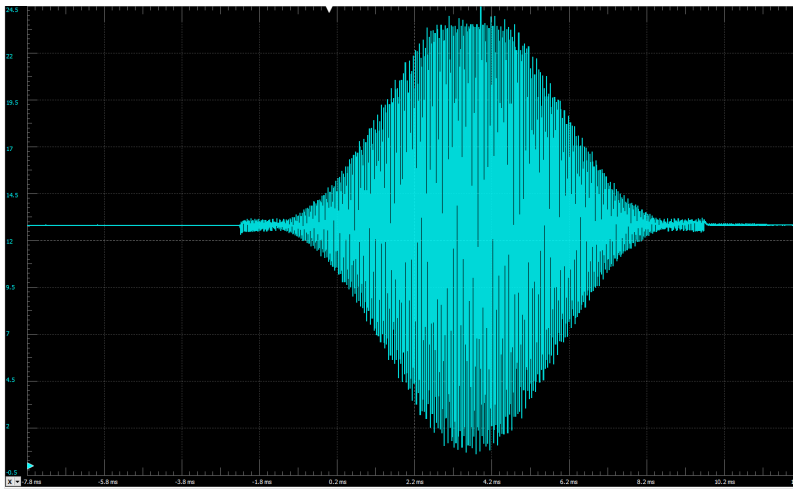

Fig. 8. Pulse Measured at the Output of the Acoustic Pinger Amplifier. Oscilloscope Configuration: Vertical Axis is the Amplitude in Volts (1.5V/div). horizontal Axis is the Time (2ms/div).

\section{Energy Detector}

The energies of the hydrophone channel can be calculated using the following formula:

$$
\text { Energy }=\sum_{n=0}^{N} y_{h}(n)^{2}
$$

Where $y_{h}$ is the received signal from the hydrophone and $N$ is the number of samples.

1) Pulse energy: The short integrator is set to measure the pulse energy during a short period. By analogy with an RC filter [26], the equation of an RC integrator is as follows:

$$
Y(n)=\frac{X(n)}{\alpha}+Y(n-1), R C_{-} \alpha=\alpha . T e
$$

The $\mathrm{z}$ transfer function of this integrator is:

$$
H(z)=\frac{1}{\alpha-(\alpha-1) \cdot Z^{-1}}
$$

This is a first order low pass filter and cutoff frequency:

$$
F_{c}=\frac{1}{2 \cdot \pi \cdot R C_{-} \alpha}
$$

The short Integrator is updated with a new energy sample as follows:

$\operatorname{Intg} C(n)=\frac{\text { Energy_antenna }-\operatorname{Intg} C(n-1)}{R C \_\alpha}+\operatorname{Intg} C(n-1)$

2) Noise energy: The long integrator aims at estimating the noise level in a long period. Similar to the short integrator, the long integrator is updated with a new energy sample as follows:

$$
\operatorname{Intg} L(n)=\frac{E n-\operatorname{Intg} L(n-1)}{R C_{-} \beta}+\operatorname{Intg} L(n-1)
$$

3) Detection contrast: The detection contrast can be then estimated:

$$
\text { Detection_Contrast }=\frac{\operatorname{Intg} C(n)}{\operatorname{IntgL(n)}}
$$

\section{E. MATLAB Simulation}

The acoustic chain and energy detector described above were implemented in MATLAB. The objective of this simulation is to qualify the capability of the designed chain to detect acoustic properly. The test signals are considered as pure tone sine waves shaped in blackman window and combined with white gaussian noise. Fig. 9 shows the frame used for the test, which is combined from pulses with $200 \mathrm{~ms}$ time-spaced simulating the reception of acoustic pulses by the transceiver.

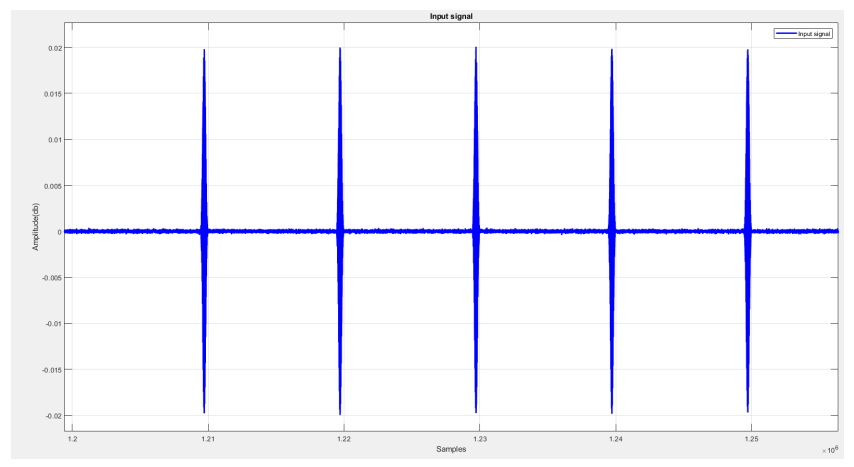

Fig. 9. Acoustic Pulses Frame Showing the form of Pulses Received by the Transceiver. SNR $=40 \mathrm{~dB}$, Frequency $=22 \mathrm{kHz}$, Amplitude $=20 \mathrm{mV}$, Pulse width $=12 \mathrm{~ms}$, Sampling Rate $=48 \mathrm{kHz}$. Horizontal Axis is the Time in Samples $(20.8 \mu \mathrm{s})$. Vertical Axis is the Amplitude in Volts.

Fig. 10 gives the received pulses pass through a low pass filter of 330 coefficients $(6.8 \mathrm{~ms})$. This filter is able to attenuate adjacent channels with up to $-80 \mathrm{~dB}$ at $500 \mathrm{~Hz}$ which allows the acoustic chain to process multi-channels pulses in the range between 18 and $22 \mathrm{kHz}$. 


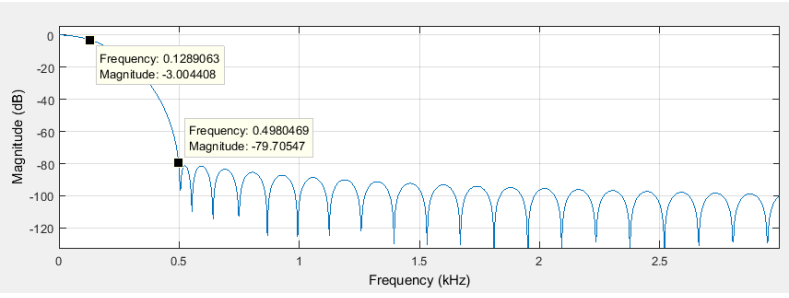

Fig. 10. Low Pass Filter Design in MATLAB. 330 Coefficients (fixed-point conversion Q21), Cutoff Frequency at -3db: $129 \mathrm{~Hz}$, Adjacent Channel Attenuation : $-80 \mathrm{~dB}$ at $500 \mathrm{~Hz}$.

Fig. 11 gives a comparison of the integrators measurements in response to the acoustic frame. In blue is the $5.33 \mathrm{~ms}$ short integrator following the shape of the pulse. The long integrator, in red, gives the estimation of noise.

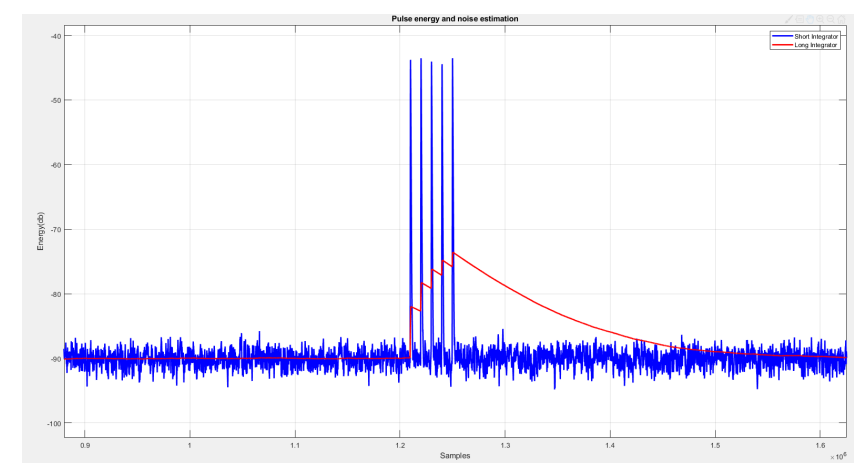

Fig. 11. Integrators Measurements. Horizontal Axis is the Time in Samples (20.8 $\mu \mathrm{s})$. Vertical Axis is the Amplitude in $\mathrm{dB}$.

The energy detection profile for the existing system (IntegL $=1366 \mathrm{~ms}$, IntegC $=5.33 \mathrm{~ms}, 12 \mathrm{~dB}$ detection threshold) is also presented in Fig. 12. The blue solid line is the $12 \mathrm{~dB}$ detection threshold. The energy detection level, in red color, $\left(=\frac{I n t g C}{I n t g L}\right)$ exceeds the detection threshold when pulses are received.

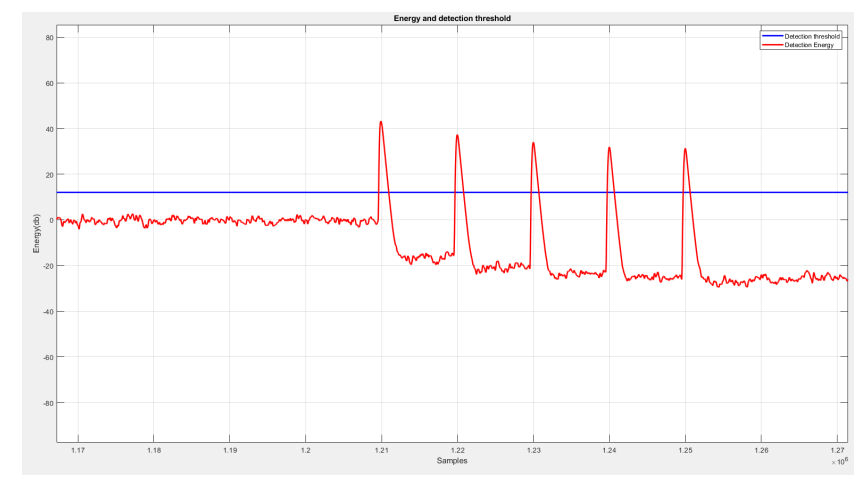

Fig. 12. Energy Detection Profile of the Acoustic Chain. Horizontal Axis is the Time in Samples $(20.8 \mu \mathrm{s})$. Vertical Axis is the Amplitude in dB.

\section{RESUlTS AND DisCUSSION}

\section{A. Sea Experiments}

This section presents two sets of experimental results. The developed system was first tested in the Marina Bouregreg harbor, located at the mouth of the Bouregreg River, on the shore of SALE, Morocco. Then we reproduced tests in Guerlédan lake, France (Fig. 13). (a)

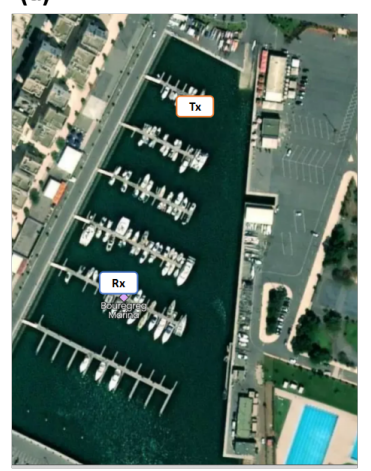

(b)

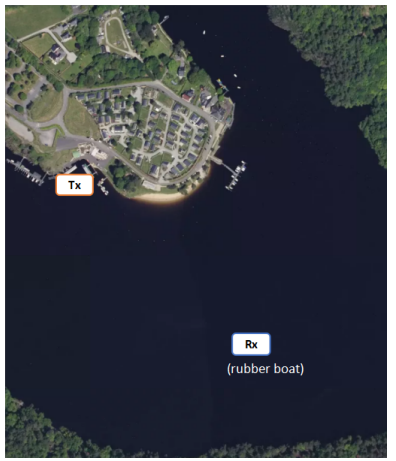

Fig. 13. Map Images Showing the Location of the Pinger (Tx) and the Transceiver (Rx) Installed in (a) Marina Harbor and (b) Guerlédan Lake.

1) Experimental platform: A schematic diagram of the experimental platform is shown in Fig. 14. The experiments reported herein rely on a transmitter electronic board that was set on the deck, while its watertight ceramic was immersed at 2 meters depth. The receiver electronic board, connected to a Laptop, was also set on the deck, with a distance to up to 280 meters far away from the transmitter. The receiver hydrophone antenna was immersed at a depth 2 meters. The transmitter and the receiver were put in line of sight. (a)

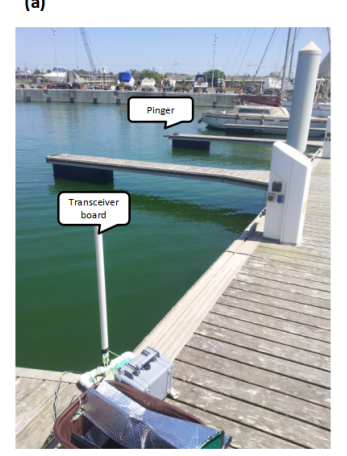

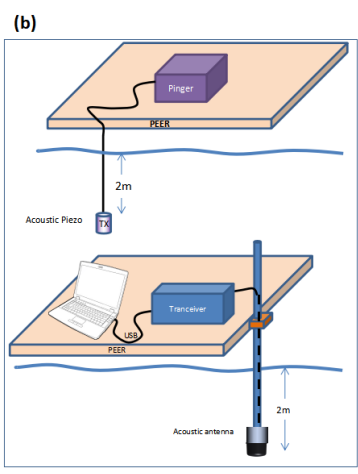

Fig. 14. (a) Image of the Experimental Platform at Marina Harbor. (b) Schematic Diagram Showing the Acoustic Pinger and the Acoustic Transceiver Boards.

2) Results: The acoustic pinger was programmed to send $20 \mathrm{kHz}$ pure tone acoustic pulses at every second. The acoustic transceiver is receiving acoustic pulses through the acoustic antenna immersed in water. The digital signal processor detects pulses (at $20 \mathrm{kHz}$ frequency) and computes the time of arrival. The Flash MCU of the transceiver reads data from DSP 
memory through HPI link and outputs the computed distance to a laptop through serial USB. The sea trial results have been obtained by first synchronizing the pinger and the transceiver by radio to have the same time reference. Then by varying the distance between the pinger and the transceiver and measuring the time-of-arrival (ToA) of acoustic pulses at the transceiver. The recorded ToAs are logged into the Laptop PC in reference to the appropriate position. The DSP firmware parameters were set to: (a) Short Integrator period of $5.33 \mathrm{~ms}$. (b) A Long Integrator period of $1.366 \mathrm{~s}$. (c) Detection threshold of $12 \mathrm{~dB}$. (d) The sound velocity was taken equal to $1500 \mathrm{~m} / \mathrm{s}$.

Fig. 15 shows an example of experiments data recorded in Guerlédan lake with pinger deployed $45 \mathrm{~m}$ far away from the transceiver. This graph shows a minimum error of $0.003 \mathrm{~m}$, a maximum error of $1.48 \mathrm{~m}$, an average error of $0.62 \mathrm{~m}$ and a peak-to-peak variance of $1.47 \mathrm{~m}$.

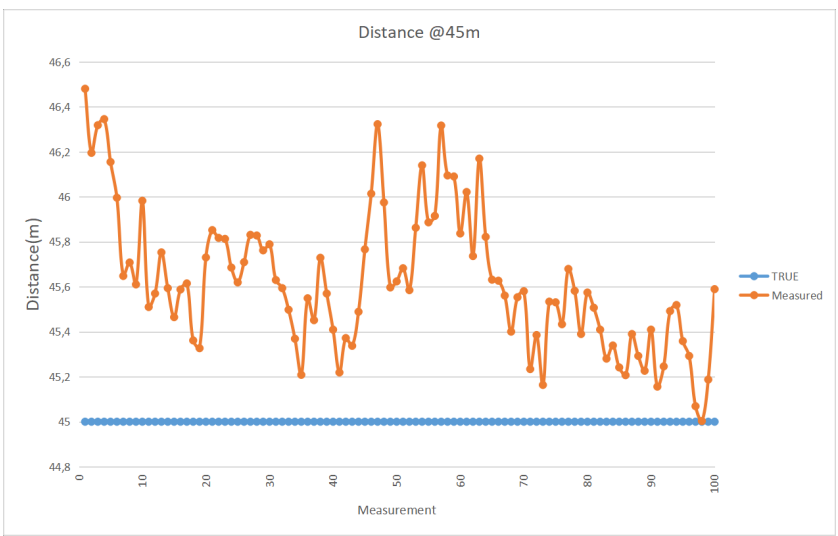

Fig. 15. Measured Distance at $45 \mathrm{~m}$. In Blue is the True Value (45m). In Orange is the Measured Distance by the Transceiver. Horizontal Axis is the Measurement Number. Vertical Axis is the Distance in Meters.

Table I summarizes the obtained performances. The detection contrast at Marina harbor conditions (low depth, noisy environment) was estimated to be very low (less than 20 $\mathrm{dB}$ at $280 \mathrm{~m})$, which explains the low performance $(520 \mathrm{~m}$ maximum range, $+/-9 \mathrm{~m}$ error at $280 \mathrm{~m})$. A better performance was obtained $(1.2 \mathrm{~km}$ detection range, $+/-4 \mathrm{~m}$ error at $300 \mathrm{~m})$ when we reproduced the same experiments in the Guerlédan lake (France) with better conditions: the detection contrast was measured around $32 \mathrm{~dB}$ at 300 meters.

TABle I. Sea Experiments Performance. Pinger Pulse Width $=$ $12 \mathrm{MS}$, SAMPLING RATE $=48 \mathrm{KHZ}$. TRANSCEIVER DETECTION THRESHOLD $=12 \mathrm{DB}$

\begin{tabular}{|c|c|c|c|}
\hline Environment & Water depth & Distance accuracy & detection range \\
\hline Marina harbor & $4 \mathrm{~m}$ & $+/-9 \mathrm{~m}$ measured at $280 \mathrm{~m}$ & $520 \mathrm{~m}$ \\
\hline Guerlédan lake & $30 \mathrm{~m}$ & $+/-4 \mathrm{~m}$ measured at $300 \mathrm{~m}$ & $1.1 \mathrm{~km}$ \\
\hline
\end{tabular}

\section{B. Improving the Noise Estimation}

1) Observation: During sea trial experiments, we have noticed that the channel noise measurements given by transceiver onboard the vehicle considerably increase with the reception of acoustic pulses from transmitters as presented in Fig. 16.

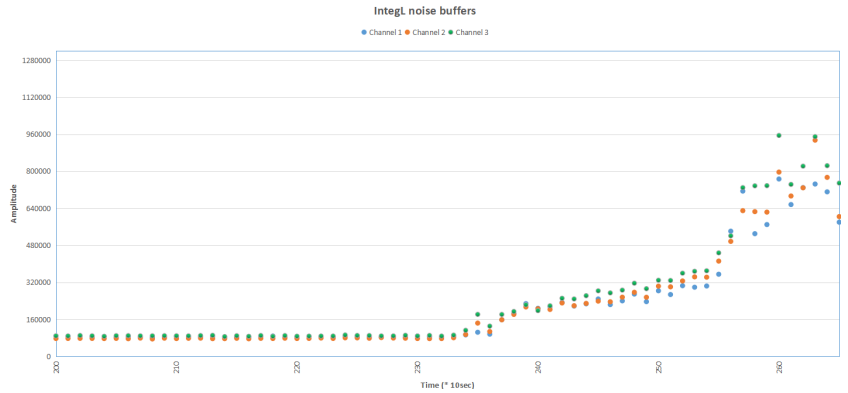

Fig. 16. Long Integrator Noise Buffer Recorded During Sea Trial Experiments at Guerlédan Lake, France. Vehicle Navigating in Free Run. Acoustic Pulses Received Every 200 ms.

This obtained records show that the presence of the signal in the acoustic chain leads to an overestimation of the noise which has a significant "blanking" effect. Furthermore, the chain has a memory that is intentionally adjusted to obtain a correct estimate of the noise ( a long memory). This phenomena can be proven using formulas in Section ??. The present context is rather favorable since the expected signal is known. We can therefore inhibit the calculation of the variance of noise during the pulse presence: (1) Either by delaying the long integration calculation quite enough to make sure the signal does not pollute the noise. (2) Or by simply freezing the long integrator at the first sign of the presence of the signal, as described in the next section.

2) Proposed solution: Refer to Fig.17 for the next discussion. The algorithm of freezing the long integrator (IntgL) is proceeded as follows: In the absence of pulses, the Long Integrator is calculated as before where the short integrator ( $\operatorname{IntgC}=5.33 \mathrm{~ms})$ is considered to check the evolution of pulses energies. If the difference between the $\operatorname{IntgC}$ and the IntgL exceeds $3 \mathrm{~dB}$, we stop feeding the IntgL. These simulations were made with an IntgL always frozen if the difference between the $\operatorname{IntgCp}$ and the $\operatorname{IntgL}$ is more than 3 $\mathrm{dB}$, and de-frozen if the difference is less than $3 \mathrm{~dB}$. Fig. 18 shows the new noise profile (Long integrator). 


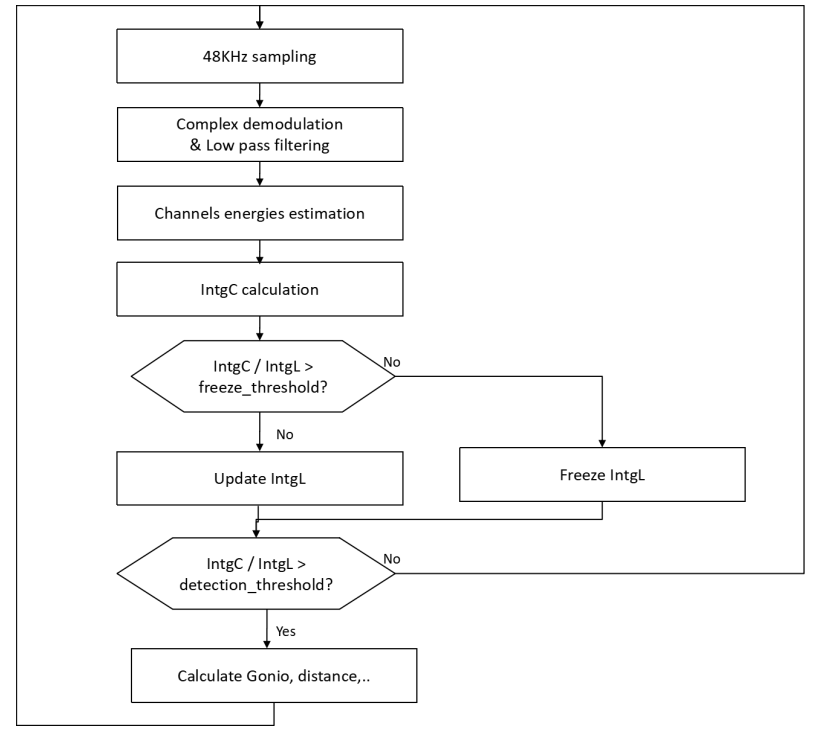

Fig. 17. Freezing IntgL Flowachart. Proposed to Remove the Sea Noise Estimation bias Caused by the Presence of the Pulses.

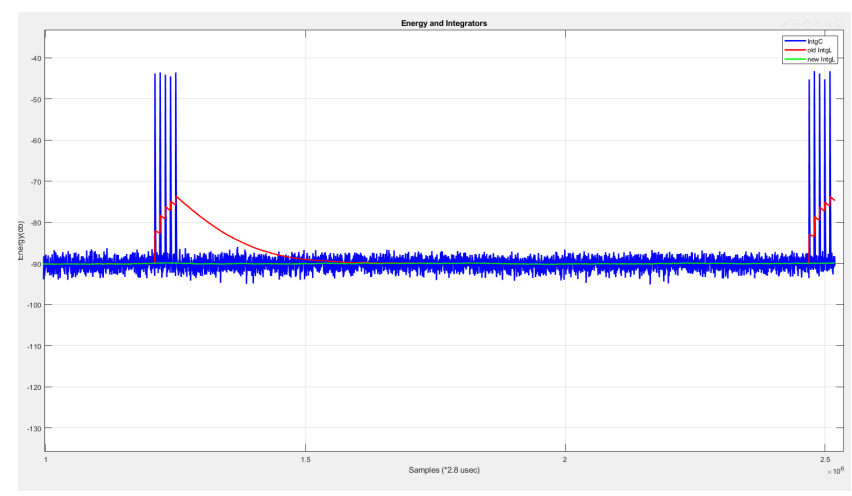

Fig. 18. Long Integrator Profile. In Blue, is the $5.33 \mathrm{~ms}$ Short Integrator. In Red, the Old Long Integrator with Value Increased in the Middle of Pulses. The New Long Integrator (in green) Maintains the Noise Level and Got Frozen when Pulses are Presents.

From the obtained results, we notice that the long integrator is sensible to the acoustic pulses reception which makes the estimation of noise level biased with an offset of more than $10 \mathrm{~dB}$ after some seconds of navigation. Means that the real detection threshold is biased with $10 \mathrm{~dB}$ and becomes $22 \mathrm{~dB}$ instead of $12 \mathrm{~dB}$. The existing design where the long integrator is fed in permanence is working well with systems receiving pulses with low frequency ( $1 \mathrm{~Hz}$ or less). Otherwise, if we receive continuous pulses every $200 \mathrm{~ms}(5 \mathrm{~Hz}$ or more), this conducts to a biased estimation of the noise level because of the high presence of pulses in noise estimation. So the way we are handling the long integrator is not optimal anymore. This was fixed by freezing the long integrator during the presence of the pulse which will increase the SNR and improve the detection range.

\section{Improving the Distance Accuracy}

As reported in the sea trial results shown in I, a limitation of the realized system appears in term of inaccuracy of ranging (up to $+/-9$ m were measured with low SNR configuration). This is explained by the fact that the existing chain is not optimized for accurate distance measurement because of the large width of the acoustic signal (12 ms). The objective of this section is to review the existing chain and propose solutions to improve its distance accuracy. Basically, decimetric accuracy on the distances is obtained by reducing the width of the acoustic pulses [27]: (1) Either by physical means, by shortening the transmission time in water or (2) by pulse compression methods (not studied in this paper). The following solutions were proposed for investigation: (1) The existing chain : First, we need to qualify the distance accuracy of the the classic processing chain with $12 \mathrm{~ms}$ blackman pulses that turns today in the transceiver. (2) An experimental processing chain: based on the existing chain, with implements a tiny square pulse of $208 \mu$ s width instead of blackman pulse. In addition to increasing the sampling rate to to $96 \mathrm{kHz}$, we will need to adapt the short integrator to this pulse width. At this stage, we will keep the low pass filtering as is. (3) A high resolution processing chain: identical to the experimental chain, but with a low pass filtering well adapted to the width of the pulse.

1) Qualification of the existing chain: MATLAB simulations have been performed using a simple frame of pure tone acoustic pulses with $2 \mathrm{~s}$ time-spaced. Each pulse is a blackman sine wave combined with white gaussian noise as shown previously in Fig. 7. The measurement of detection ToA was performed by varying the energy of the input signal from high SNR (more than $100 \mathrm{~dB}$ ) to low SNR $(12 \mathrm{~dB})$. An example showing the ToA of an acoustic pulse of $67 \mathrm{~dB}$ is illustrated in Fig. 19. The status of detection goes high when the contrast (difference between the pulse energy and the noise) reaches threshold of $12 \mathrm{~dB}$.

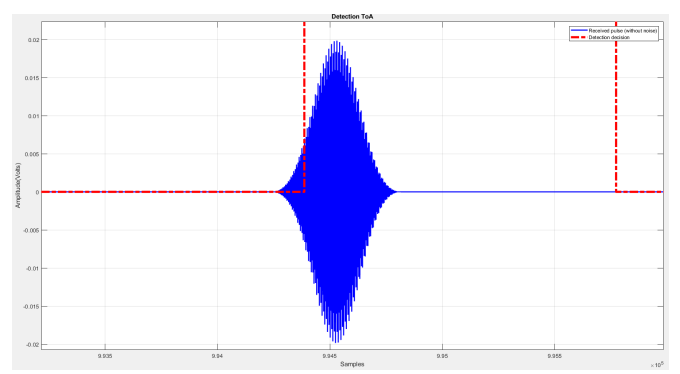

Fig. 19. Detection ToA Measurement with the Existing Chain. In Blue is the Acoustic Pulse. In Red is the Status of Detection $(0=$ no detection, $1=$ detection). Blackman Pulse width $=12 \mathrm{~ms}$, Sampling Rate $=48 \mathrm{Khz}$, Short Integrator $=6 \mathrm{~ms}$, Low Pass Filter $=330$ Coefficients. Horizontal Axis is the Time in Samples $(20.8 \mu$ s). Vertical Axis is the Amplitude in Volts.

The detection ToA and delays measurements, summarized in Table II, were obtained depending the variation of the detection contrast (SNR). The measurements show that the detection delay depends on the contrast level : with high SNR, the detection is at the beginning of the pulse, while the detection time is delayed to up to $8.916 \mathrm{~ms}(13,375 \mathrm{~m}$ distance accuracy) at the limit of detection $(12 \mathrm{~dB})$. 
TABLE II. Existing Chain Detection Delay Measured as FUNCTION OF THE CONTRAST. BLACKMAN PULSE WIDTH $=12 \mathrm{MS}$, SAMPLING RATE $=48 \mathrm{KHZ}$, SHORT INTEGRATOR $=6 \mathrm{MS}$, LOW PASS FILTER $=330$ COEFFICIENTS

\begin{tabular}{|c|c|c|}
\hline Contrast $(\mathrm{dB})$ & Detection delay $(\mathrm{ms})$ & Distance accuracy $(\mathrm{m})$ \\
\hline more than $100 \mathrm{~dB}$ & reference & - \\
\hline $87 \mathrm{~dB}$ & $1,458 \mathrm{~ms}$ & $2,187 \mathrm{~m}$ \\
\hline $67 \mathrm{~dB}$ & $2,354 \mathrm{~ms}$ & $3,531 \mathrm{~m}$ \\
\hline $47 \mathrm{~dB}$ & $3,583 \mathrm{~ms}$ & $5,374 \mathrm{~m}$ \\
\hline $27 \mathrm{~dB}$ & $5,625 \mathrm{~ms}$ & $8,437 \mathrm{~m}$ \\
\hline $17 \mathrm{~dB}$ & $7,354 \mathrm{~ms}$ & $11,031 \mathrm{~m}$ \\
\hline $12 \mathrm{~dB}$ & $8,916 \mathrm{~ms}$ & $13,375 \mathrm{~m}$ \\
\hline
\end{tabular}

2) Qualification of the experimental processing chain: Similar to the qualification of the existing chain, the pulse frame contains acoustic pulses with 2 seconds time-spaced. However, the format of the pulse was to square sine wave instead of blackman, and the pulse width was reduced to 208 $\mu$ s instead of $12 \mathrm{~ms}$. Fig. 20 illustrates the pulse format used and a ToA measurement when the SNR is around $82 \mathrm{~dB}$.

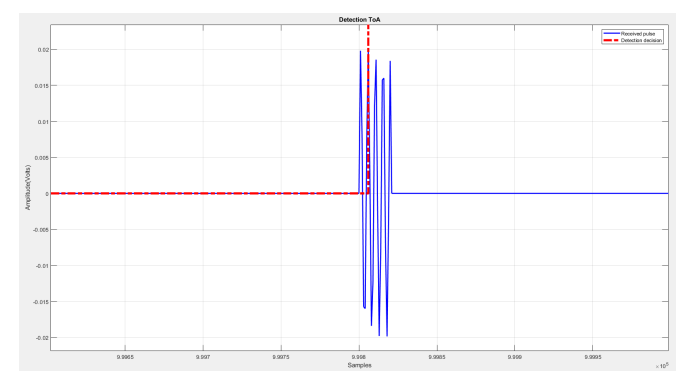

Fig. 20. Detection ToA with the Experimental Processing Chain. In Blue is the Acoustic Pulse. In Red is the Status of Detection $(0=$ no detection, $1=$ detection). Square Sine Pulse width $=208 \mu \mathrm{s}$, Sampling Rate $=96 \mathrm{Khz}$, Short Integrator $=96 \mu \mathrm{s}$, Low Pass Filter $=330$ Coefficients $(3.4 \mathrm{~ms})$. Horizontal Axis is the time in Samples $(10.4 \mu \mathrm{s})$. Vertical Axis is the Amplitude in Volts.

The detection ToA and delays of the experimental processing chain were measured depending the variation of the detection contrast level. The obtained results are presented in Table III. The measurements show that the maximum detection delay was reduced to up to $1.145 \mathrm{~ms}$ (around $1,718 \mathrm{~cm}$ distance accuracy compared to $13,375 \mathrm{~m}$ obtained previously with the existing processing chain).

TABLE III. Experimental Processing Chain Detection Delay Measured as Function of the Detection Contrast. SQuare Sine PULSE WIDTH $=208 \mu \mathrm{s}$, SAMPLING RATE $=96 \mathrm{KHZ}$, SHORT INTEGRATOR $=96 \mu \mathrm{s}$, LOW PASS FILTER $=330$ COEFFICIENTS (3.4 MS). HORIZONTAL

AXIS IS THE TIME IN SAMPLES $(10.4 \mu \mathrm{S})$. VerTICAL AXIS IS THE AMPLITUDE IN VOLTS

\begin{tabular}{|c|c|c|}
\hline Contrast $(\mathrm{dB})$ & Detection delay $(\mathrm{ms})$ & Distance accuracy $(\mathrm{m})$ \\
\hline more than $100 \mathrm{~dB}$ & reference & - \\
\hline $82 \mathrm{~dB}$ & $0,052 \mathrm{~ms}$ & $0,078 \mathrm{~m}$ \\
\hline $62 \mathrm{~dB}$ & $0,187 \mathrm{~ms}$ & $0,281 \mathrm{~m}$ \\
\hline $42 \mathrm{~dB}$ & $0,437 \mathrm{~ms}$ & $0,656 \mathrm{~m}$ \\
\hline $22 \mathrm{~dB}$ & $0,822 \mathrm{~ms}$ & $1,234 \mathrm{~m}$ \\
\hline $12 \mathrm{~dB}$ & $1,145 \mathrm{~ms}$ & $1,718 \mathrm{~m}$ \\
\hline
\end{tabular}

3) Qualification of the high resolution processing chain: Now, with the high resolution processing chain, we are going to adapt the filter window to the tiny pulse. By analogy to the low pass filter of the existing chain, the filter window was taken as the half of pulse width $\left(\frac{208}{2}=104 \mu \mathrm{s}\right)$. Which is set with 10 coefficients at $96 \mathrm{KHz}$ sampling rate. Fig. 21 illustrates the low pass filter profile in MATLAB Filter Design Tool [28]. This filter is of course not very selective and not robust against adjacent channels, nevertheless it allows to reject out-of-band noises. Therefore, the high resolution processing chain will be limited to one-channel instead of eight.

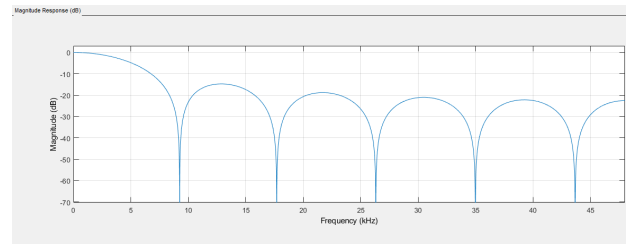

Fig. 21. Low Pass Filter Simulated with the High Resolution Processing Chain. 10 Coefficients FIR. Sampling Rate $=96 \mathrm{Khz}$. Rejection $-15 \mathrm{~dB}$ at 7 $\mathrm{KHz}$.

The detection ToA and delays of the high resolution processing chain were measured depending the variation of the contrast level. The obtained results are presented in Table IV. A very good performance was obtained with a maximum detection delay of $72.91 \mu$ s (around $10,93 \mathrm{~cm}$ distance accuracy) at the limit of detection $(12 \mathrm{~dB})$.

TABLE IV. One-ChanNel High Resolution Processing Chain Detection Delay Measured as Function of the Detection Contrast. Square Sine Pulse Width $=208 \mu \mathrm{s}$, SAmpling RATE $=96$ $\mathrm{KHz}$, SHORT INTEGRATOR $=96 \mu \mathrm{s}$, LOW PASS FILTER $=10$ CoEfFicients $(104 \mu \mathrm{s})$

\begin{tabular}{|c|c|c|}
\hline Contrast $(\mathrm{dB})$ & Detection delay $(\mathrm{ms})$ & Distance accuracy $(\mathrm{m})$ \\
\hline more than $100 \mathrm{~dB}$ & reference & - \\
\hline $86 \mathrm{~dB}$ & $0 \mathrm{~ms}$ & $0 \mathrm{~m}$ \\
\hline $66 \mathrm{~dB}$ & $0 \mathrm{~ms}$ & $0 \mathrm{~m}$ \\
\hline $46 \mathrm{~dB}$ & $0 \mathrm{~ms}$ & $0 \mathrm{~m}$ \\
\hline $26 \mathrm{~dB}$ & $0,052083 \mathrm{~ms}$ & $0,03124 \mathrm{~m}$ \\
\hline $12 \mathrm{~dB}$ & $0,072916 \mathrm{~ms}$ & $0,1093 \mathrm{~m}$ \\
\hline
\end{tabular}

\section{CONClusions}

In this paper, we presented the design of device able to detect acoustic signals underwater. We built prototypes that can be mounted onboard AUVs allowing them to detect and find targets that are sending acoustic waves. The proposed hardware architecture of the pinger is based on an ultra-low-power Flash Micro-controller sending pure tone acoustic pulses at narrow band frequency. The transceiver hardware onboard the AUVs incorporates a Digital Signal Processor implementing energy based spectrum sensing mechanism to detect the acoustic pulses sent by the pinger.

The acoustic chain was designed to process pure tone sine waves shaped in $12 \mathrm{~ms}$ blackman window at $48 \mathrm{kHz}$ sampling rate, $6 \mathrm{~ms}$ short integrator and 330 coefficients $(6.8$ $\mathrm{ms}$ ) of low pass filter. In addition, it supports multi-channels operation with the capability to recognize pulses from eight different pingers transmitting at different frequencies. The time-of-arrival of incoming pulses is then measured and the pinger position can be estimated. 
Experiments with the realized system were carried out at sea with two different configurations: (a) at Marina harbor (considered as noisy environment), a very shallow waters (maximum depth $; 5 \mathrm{~m}$ ) where the transceiver was able to detect pingers at $520 \mathrm{~m}$ range, (b) at Guerlédan lake (considered as clean environment) with up to $30 \mathrm{~m}$ depth where more than 1 $\mathrm{km}$ range was obtained. During sea trials, two limitations of were identified: (1) The noise estimator was biased with more than $10 \mathrm{~dB}$ in the case where the receiver detects continuous pulses with a rate of $200 \mathrm{~ms}$ or less. We fixed this issue by freezing the long integrator at the beginning of pulse detection, which removed the bias and improved the detection range. (2) The distance accuracy was evaluated around $2 \mathrm{~m}$ in high SNR and $14 \mathrm{~m}$ at limit of detection. This performance can be improved to around $10 \mathrm{~cm}$ by using the one-channel high resolution processing chain with tiny square pulses of 208 $\mu$ s processed at $96 \mathrm{kHz}$ sampling rate. As a consequence, the system will loose the feature of multi-channels operation. For future works, pulse compression technique should be investigated with matched filtering in order to enhance the performance of our system, especially in noisy environments.

\section{ACKNOWLEDGMENT}

This work was supported by Arkeocean SARL, France. The authors would like to thank the Marina harbor office who supported the sea trials of this work.

\section{REFERENCES}

[1] Yao Yao. Cooperative navigation system for multiple unmanned underwater vehicles. IFAC Proceedings Volumes, 46(20):719-723, 2013. 3rd IFAC Conference on Intelligent Control and Automation Science ICONS 2013

[2] Ian F. Akyildiz, Dario Pompili, and Tommaso Melodia. Underwater acoustic sensor networks: research challenges. Ad Hoc Networks, 3(3):257-279, 2005.

[3] Archana Toky, Rishi Pal Singh, and Sanjoy Das. Localization schemes for underwater acoustic sensor networks - a review. Computer Science Review, 37:100241, 2020.

[4] P. Rizzo. 17 - sensing solutions for assessing and monitoring underwater systems. In M.L. Wang, J.P. Lynch, and H. Sohn, editors, Sensor Technologies for Civil Infrastructures, volume 56 of Woodhead Publishing Series in Electronic and Optical Materials, pages 525-549. Woodhead Publishing, 2014.

[5] Imane Salhi, Martyna Poreba, Erwan Piriou, Valerie Gouet-Brunet, and Maroun Ojail. Chapter 8 - multimodal localization for embedded systems: A survey. In Michael Ying Yang, Bodo Rosenhahn, and Vittorio Murino, editors, Multimodal Scene Understanding, pages 199278. Academic Press, 2019.

[6] B Mishachandar and S Vairamuthu. An underwater cognitive acoustic network strategy for efficient spectrum utilization. Applied Acoustics, 175:107861, 2021.

[7] David Munoz, Frantz Bouchereau, Cesar Vargas, and Rogerio Enriquez. Chapter 1 - the position location problem. In David Munoz, Frantz Bouchereau, Cesar Vargas, and Rogerio Enriquez, editors, Position Location Techniques and Applications, pages 1-22. Academic Press, Oxford, 2009.

[8] L. Bjorno. Chapter 14 - underwater acoustic measurements and their applications. In Thomas H. Neighbors and David Bradley, editors, Applied Underwater Acoustics, pages 889-947. Elsevier, 2017.

[9] N. Crasta, D. Moreno-Salinas, A.M. Pascoal, and J. Aranda. Multiple autonomous surface vehicle motion planning for cooperative rangebased underwater target localization. Annual Reviews in Control, 46:326-342, 2018.
[10] S. Longhi, A. Monteriù, and M. Vaccarini. Cooperative control of underwater glider fleets by fault tolerant decentralized mpc. IFAC Proceedings Volumes, 41(2):16021-16026, 2008. 17th IFAC World Congress.

[11] Jian Lu, Xu Chen, Maoxin Luo, and Yanran Zhou. Cooperative localization for multiple auvs based on the rough estimation of the measurements. Applied Soft Computing, 91:106197, 2020.

[12] Shaonan Li, Wenyu Qu, Chunfeng Liu, Tie Qiu, and Zhao Zhao. Survey on high reliability wireless communication for underwater sensor networks. Journal of Network and Computer Applications, 148:102446, 2019.

[13] Lin Ma, T. Aaron Gulliver, Anbang Zhao, Chunsha Ge, and Xuejie Bi. Underwater broadband source detection using an acoustic vector sensor with an adaptive passive matched filter. Applied Acoustics, 148:162$174,2019$.

[14] Shuxia Huang, Shiliang Fang, and Ning Han. Iterative matching-based parameter estimation for time-scale underwater acoustic multipath echo. Applied Acoustics, 159:107094, 2020.

[15] Daniela Mercedes Martínez Plata and Ángel Gabriel Andrade Reátiga. Evaluation of energy detection for spectrum sensing based on the dynamic selection of detection-threshold. Procedia Engineering, 35:135143, 2012. International Meeting of Electrical Engineering Research 2012.

[16] B. Sarala, S. Rukmani Devi, and J. Joselin Jeya Sheela. Spectrum energy detection in cognitive radio networks based on a novel adaptive threshold energy detection method. Computer Communications, 152:17, 2020.

[17] L. KOPP. Détection et estimation en traitement d'antenne : théorie. Techniques de l'ingénieur Systèmes radars, TIB591DUO(te5225), 2003.

[18] L. Kopp, G. Bienvenu, and M. Aiach. New approach to source detection in passive listening. In ICASSP '82. IEEE International Conference on Acoustics, Speech, and Signal Processing, volume 7, pages 779-782, 1982.

[19] Marco Gori. Chapter 5 - deep architectures. In Marco Gori, editor, Machine Learning, pages 236-338. Morgan Kaufmann, 2018.

[20] Dogan Ibrahim. Chapter 5 - pic18 microcontroller development tools. In Dogan Ibrahim, editor, SD Card Projects Using the PIC Microcontroller, pages 257-297. Newnes, Boston, 2010.

[21] Robert Oshana. 5 - dsp architectures. In Robert Oshana, editor, DSP Software Development Techniques for Embedded and Real-Time Systems, Embedded Technology, pages 123-158. Newnes, Burlington, 2006.

[22] John H. Davies. Chapter 2 - the texas instruments msp430. In John H. Davies, editor, MSP430 Microcontroller Basics, pages 21-42. Newnes, Burlington, 2008.

[23] Redouane Es-sadaoui, Lahoucine Azergui, Youssef Ghanam, and Jamal Khallaayoune. Design and experimentation of a low-power iot embedded system for wireless underwater sensing. In International Conference on Wireless Networks and Mobile Communications, WINCOM 2017, Rabat, Morocco, November 1-4, 2017, pages 1-6. IEEE, 2017.

[24] Jinwang Yi, Diba Mirza, Ryan Kastner, Curt Schurgers, Paul Roberts, and Jules Jaffe. Toa-ts: Time of arrival based joint time synchronization and tracking for mobile underwater systems. Ad Hoc Networks, 34:211223, 2015. ADVANCES IN UNDERWATER COMMUNICATIONS AND NETWORKS.

[25] W. Kenneth Jenkins, Douglas L. Jones, and Bill J. Hunsinger. 28 discrete-time signal processing. In Wendy M. Middleton and Mac E. Van Valkenburg, editors, Reference Data for Engineers (Ninth Edition), pages 28-1-28-39. Newnes, Woburn, ninth edition edition, 2002.

[26] Marc T. Thompson. Chapter 2 - review of signal processing basics. In Marc T. Thompson, editor, Intuitive Analog Circuit Design (Second Edition), pages 15-52. Newnes, Boston, second edition edition, 2014.

[27] M.A. Do. Modern methods of improving the range accuracy of ctfm sonars. Ultrasonics, 22(3):110-114, 1984.

[28] Ibrahim Abdulhadi Sulaiman, Hussain Mohammad Hassan, Mohammad Danish, Munendra Singh, P.K. Singh, and Manisha Rajoriya. Design, comparison and analysis of low pass fir filter using window techniques method. Materials Today: Proceedings, 2020. 